\title{
On the minimum number of general or dedicated controllers required for system controllability
}

\author{
Alberto Portal, Pedro J. Zufiria
}

\begin{abstract}
A B S T R A C T
The paper determines the minimum number of general or dedicated controllers which are required to guarantee the controllability of a class of dynamical systems. For this purpose, we begin by reformulating Kalman controllability condition for linear systems $(A, B)$ in the context of the similarity equivalence class associated with $A$, and then we provide new alternative characterizations of such controllability condition based on the cases when matrix $A$ is in Jordan or Frobenius form, so that theoretical aspects and computational requirements are comparatively commented. In the second part of the paper, given a system state matrix $A$, we solve the optimal design problem of determining the minimum number of controllers (columns of $B$ ) required for making $(A, B)$ controllable, resorting also to the Jordan and Frobenius canonical forms in the similarity equivalence class associated with $A$. These canonical forms are proven to be also fundamental to provide bounds on the minimum number of dedicated controllers (corresponding to columns of $B$ with a single non-zero element) required to make $(A, B)$ controllable. Some examples serve to illustrate the fundamental results.
\end{abstract}

\section{Introduction}

Controllability and observability of dynamical systems which evolve under the influence of external inputs and which allow access to measurable outputs are two fundamental issues when addressing the design of control systems [1-4]. Controllability characterizes the possibility to move, by injecting a feasible external input, the internal state of the system from any initial state to any other final state in a finite time interval [5]. On the other hand, observability characterizes the possibility of determining the internal state of the system based on the measurement of accessible variables (outputs) during a finite time interval. Both problems are dual and they have been thoroughly studied in the literature where the so called controllability and observability conditions are well defined, specially for linear systems [5-7]. In this paper we address optimality issues in the controller design for linear time-invariant systems, the obtained results being directly translatable to the dual system observer design problem.

Standard control theory has been developed under the assumption that the system equations (corresponding to its dynamics) and/or the actuator restrictions (determining the feasible external inputs) are given a priori. In this context, the controllability condition [8] serves as a test on the existence of input control laws having some desirable properties. In 
the first part of this paper we reformulate such controllability condition in the context of the similarity equivalence class associated with $A$. This allows new alternative characterizations of such condition based on the cases when matrix $A$ is in Jordan or Frobenius form. Besides their advantageous computational implications, these characterizations will become relevant when addressing the subsequent optimal design problem.

Many real applications and design problems in several fields such as distributed systems [9-11] and complex networks $[12,13]$ consider the possibility of arbitrarily selecting the number and/or the location of the actuators. In these contexts, the control matrix $B$ becomes a design element, and the controllability condition serves as a design criterion. Precisely the second part of this paper addresses the optimal design problem of determining the minimum number of controllers in $B$ required for making $(A, B)$ controllable. This problem is solved making use of the Jordan and Frobenius canonical forms in the similarity equivalence class associated with $A$. Finally, these canonical forms are proven to be also fundamental to provide bounds on the minimum number of dedicated controllers which are required in matrix $B$ to make $(A, B)$ controllable.

The paper is organized as follows. Section 2 presents the fundamental controllability condition and derives specific alternative formulations for the case of $A$ in Jordan and Frobenius canonical forms; in addition, some theoretical aspects and computational requirements are commented and one example is provided. Section 3 solves the problem of determining the minimum number of required controllers in $B$ for making $(A, B)$ controllable. The problem of determining bounds on the number of required dedicated controllers is addressed in Section 4. Finally, concluding remarks are outlined in Section 5.

\section{The controllability problem}

Let us consider the linear time invariant dynamical system

$$
\frac{d v}{d t}=A x(t)+B u(t)
$$

where $x(t) \in \mathbb{R}^{n}$ and $u(t) \in \mathbb{R}^{p}$, so that $B \in \mathbb{R}^{n \times p}$. This type of systems have been extensively studied from the classical control theory perspective [5-7].

\subsection{Controllability condition}

A system is controllable if we can lead it to any final state $x_{f}$ from any initial state $x_{0}$ in finite time (1.e., in an interval [0, T]).

This controllability condition for system (1) is stated as follows (see [7] for details): the system (1) is controllable (i.e., the pair $(A ; B)$ is controllable) if and only if the controllability matrix

$$
C(A, B)=\left[B A B \cdots A^{n-1} B\right]
$$

satisfies the condition [8]:

$$
\operatorname{rank} C(A, B)=n \text {. }
$$

Based on the computational cost for determining the rank of a matrix $[14,15]$, condition (2) for general matrices $A$ and $B$ requires $\mathcal{O}\left(p \cdot n^{\omega}\right)$ operations where $\omega<2.373$ is the matrix multiplication exponent. Hence, for large systems (2) may have a limited direct applicability in the selection or design of appropriate $B$ matrices which guarantee system controllability. In the following, the analysis of canonical forms which decompose the whole state space dynamics into smaller invariant subspaces will prove to be very useful for a simpler characterization of the system controllability.

\subsection{Controllability and similarity transformations}

Given system (1), one can perform a change of coordinates defined by a similarity matrix $P \in \mathbb{C}^{n \times n}$ so that the pair $(A, B)$ becomes $\left(P^{-1} A P, P^{-1} B\right)$. Then we have:

Lemma 1. The pair $(A, B)$ is controllable if and only if $\left(P^{-1} A P, P^{-1} B\right)$ is also a controllable pair.

Proof. Since $P$ is regular, the controllability matrix $C\left(P^{-1} A P, P^{-1} B\right)$ has the same rank as $C(A, B)=P C\left(P^{-1} A P, P^{-1} B\right)$.

Whenever a matrix $\ddot{A} \in \mathbb{C}^{n \times n}$ can be written as $\ddot{A}=P^{-1} A P$ for some regular matrix $P$, we say that $\ddot{A}$ is similar to $A$ and denote $\dot{A} \sim A$ the corresponding equivalence relation. Lemma 1 provides a way to check the controllability of a system with state matrix $A$ by resorting to a controllability analysis of systems with state matrices in canonical forms which are similar to $A$.

\subsubsection{Canonical forms}

Let us denote $S_{A}=\left\{\ddot{A} \in \mathbb{C}^{n \times n}: \ddot{A} \sim A\right\}$ the set of all matrices which are similar to $A$; it is known that all matrices in $S_{A}$ have the same characteristic polynomial, hence the same set of eigenvalues. Several canonical forms can be defined within $S_{A}[16-21]$ which, under the controllability assumption, have been employed for control system analysis and design. Among them, two canonical forms will prove to be very useful due to their specific structure for controllability characterization: the 
Jordan form, which reflects maximal decomposition of the space into cyclic subspaces, and the Frobenius form for which such decomposition into cyclic subspaces is minimal.

Jordan canonical form. A matrix $J \in S_{A}$ is in Jordan canonical form if

$$
J=\bigoplus_{k=1}^{K} \bigoplus_{m=1}^{M_{k}} J_{k, m} .
$$

where $J_{k, 1}, \ldots, J_{k} M_{k}$ are the $M_{k}$ Jordan blocks associated with each $\lambda_{k} \in\left\{\lambda_{1}, \lambda_{2}, \ldots, \lambda_{k}\right\}$, the set of different eigenvalues of $A$. We will denote $n_{k m} \in \mathbb{N}$ the size of each $J_{k m}$, and we will assume, without loss of generality, that the eigenvalues are ordered so that $M=M_{1} \geq M_{2} \geq \ldots \geq M_{K}$ and for each $1 \leq k \leq K, n_{k .1} \geq n_{k .2} \geq \cdots \geq n_{k_{n, \ldots}}$.

Frobenius or rational canonical form. A matrix $F \in S_{A}$ is in Frobenius canonical form if

$$
F_{1}=\bigoplus_{m=1}^{M} F_{m} .
$$

where for $1 \leq m \leq M$ each matrix $F_{m}$ is the companion matrix associated with the polynomial $q_{m}(x)=\prod_{k=1}^{K_{m}}\left(x-\lambda_{k}\right)^{n_{k, m}}$ with $K_{m}$ denoting the number of eigenvalues of $F$ which have, at least, $m$ Jordan blocks (note that, by definition, it is $\left.K_{1} \geq K_{2} \geq \cdots \geq K_{M}\right)$, so that the following divisibility condition holds:

$$
q_{M}(x)\left|q_{M-1}(x)\right| \cdots\left|q_{2}(x)\right| q_{1}(x),
$$

since for $1 \leq k \leq K$, it is $n_{k .1} \geq n_{k .2} \geq \cdots \geq n_{k M_{k}}$.

Let us define $n_{* m}=\sum_{n=1}^{k, \cdots} n_{k . m}$ and $n_{k *}=\sum_{m=1}^{k_{k}} n_{k . m}$, so that each $q_{m}(x)$ has degree $n_{*_{m}}$ and the characteristic polynomial of $F$ is $q(x)=\prod_{m=1}^{M} q_{m}(x)=\prod_{k=1}^{K}\left(x-\lambda_{k}\right)^{n_{k+}}$. Note that if $J \sim F$, then $M$ takes the same value in (3) and (4).

Depending on the specific structure of matrix $A$, the general condition (2) for guaranteeing system controllability can be reformulated as a set of more specific conditions to be satisfied by matrix $B$. In the following section we characterize the conditions to be satisfied by matrix $B$ when $A$ displays either the Jordan or the Frobenius canonical form.

\subsection{Controllability conditions for canonical forms}

We start presenting some useful definitions regarding the submatrices which can be derived from $B$ :

Definition 1. Consider $B \in \mathbb{C}^{n \times p}$ whose jth column is denoted by $B_{j}$. Let $C=\left\{c_{1}, c_{2} \ldots c_{\ell}\right\} \subseteq\{1,2 \ldots p\}$ represent a selection of columns of $B$, so that we denote the matrix obtained from $B$ by selecting those columns indexed by $C$ as

$$
\left.B\right|_{C}=\left(\begin{array}{llll}
B_{c_{1}} & B_{c_{2}} & \cdots & B_{c_{l}}
\end{array}\right) .
$$

Hence, $\left.B\right|_{C}$ is obtained from $B$ by selecting its $C$ th columns. Analogously, denoting the ith row of $\bar{B}$ by $B^{\prime}$, let $R=$ $\left\{r_{1}, r_{2}, \ldots, r_{q}\right\} \subseteq\{1,2, \ldots, n\}$ represent a selection of rows of $B$, so that we denote the matrix obtained from $B$ by selecting those rows indexed by $R$ as

$$
\left.B\right|^{R}=\left(\begin{array}{llll}
B^{r_{1}{ }^{\top}} & B^{r_{2}} & \ldots & B^{r_{q}{ }^{\top}}
\end{array}\right)^{\top} .
$$

and we say that $\left.B\right|^{\kappa}$ is obtained from $B$ by selecting its Rth rows. The meaning of $\left.B\right|_{C} ^{K}$ is, as one may expect, $\left.\left(\left.B\right|_{C}\right)\right|^{R}=\left.\left(\left.B\right|^{R}\right)\right|_{C}$. form.

The following subsections address the cases where system matrix $A$ displays either a Jordan or a Frobenius canonical

\subsubsection{System matrix A in Jordan canonical form}

Let us consider the control system (1) with system matrix $A=J$ already in Jordan form. Hence, the controllability condition for the control system (1) boils down to the following theorem [6]:

Theorem 1. Let $J$ be as defined in (3) and $B \in \mathbb{C}^{n \times p}$. For $1 \leq k \leq K$, let $C_{k}$ be the selection of columns of $J$ corresponding to the first column of each Jordan block $J_{k . m}$. Then $\operatorname{rank} C(J . B)=n$ if and only if matrix $B$ satisfies that

$$
\left.\operatorname{rank} B\right|^{C_{k}}=M_{k}, \quad \text { for } 1 \leq k \leq K \text {. }
$$

A useful reformulation of the above Theorem 1 is the following:

Corollary 1. Let $J$ be as defined in (3) and $B \in \mathbb{C}^{n \times p}$. Define $C_{k}$ as in Theorem 1. Then, rank $C(J, B)=n$ if and only for each $k \in\{1 \ldots . . K\}$ there exists a selection $D_{k}$ of $M_{k}$ columns of matrix $B$ which satisfies

$$
\left.\operatorname{razhk} B\right|_{D_{k}} ^{C_{k}}=M_{k} .
$$

Proof. If $\operatorname{rank} \mathcal{C}(J \cdot B)=n$. Theorem 1 ensures that

$$
\left.\operatorname{rank} B\right|^{C_{k}}=M_{k} \text {, }
$$

so a selection $D_{k}$ of $M_{k}$ columns of $\left.B\right|^{C_{k}}$ such that $\left.\operatorname{rank} B\right|_{D_{k}} ^{C_{k}}=M_{l_{k}}$ can be found. 
On the other hand, if (7) holds for $1 \leq k \leq K$, consider $D=\bigcup_{k=1}^{r} D_{k}$. Then the matrix $\left.B\right|_{D}$ satisfies condition (6) and so does $B$.

Note that the applicability of (6) relies on the computability of the Jordan canonical form. For a discussion on the limitations when computing such canonical form see [22-24].

In [6] some alternative conditions for a general pair $(A, B)$ to be controllable are also stated, but they require the knowledge of the eigenvalues of matrix $A$. In the following subsection we propose an alternative controllability characterization which gets round the need of knowing such eigenvalues.

\subsubsection{System matrix A in Frobenius canonical form}

We consider now the case when the control system (1) has system matrix $A=F$ already in the Frobenius form given in (4). $F$ is similar to the following matrices (the first one, $J$, being the Jordan form and the second one, $J$, being a suitable form obtained by rearranging the blocks of $J$ ):

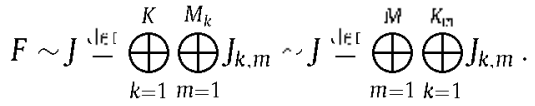

Since $J_{k m}$ is the $m$ th Jordan block associated with $\lambda_{k}$, an eigenvalue of $F_{m}$, we have that $F_{m} \sim \bigoplus_{k-1}^{K_{m}} J_{k \cdot m}=J_{m}$ for $1 \leq m \leq M$.

In order to determine a similarity transformation matrix, we now define the square matrices $W_{m}(x)$, for $1 \leq m \leq M$, as follows:

$$
\left[W_{m}(x)\right]_{1,1}- \begin{cases}\left(\begin{array}{l}
1 \\
i-1
\end{array}\right) x^{\prime-1} & \text { if } i \leq j . \\
0 & \text { if } i>j .\end{cases}
$$

whose order is that of $F_{m}$. Defining also $\left[n_{k m}\right]=\left\{1,2, \ldots, n_{k m}\right\}$ for any $n_{k m} \in \mathrm{I}$, we have the following result:

Lemma 2. Matrix

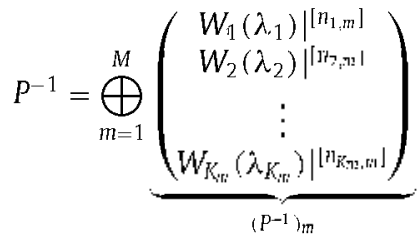

satisfies that $F=P J P^{-1}$.

Proof. Note that $\left(P^{-1}\right)_{m}$, the $m$ th block of block-diagonal matrix $P_{-}^{-1}$, is equal to $P_{m}^{1}$ which takes the form $P_{m}^{-1}=\mathcal{C}\left(j_{m}, b_{m}\right)$ for a binary vector $b_{m}$ with unitary values in the $C_{k}$ indexes (as defined in Theorem 1) and zero elsewhere (note that $b_{m}$ makes the pair $\left(J_{m}, b_{m}\right)$ controllable and hence $P_{m}^{-1}$ regular). Then, for $1 \leq m \leq M$ we need to prove that $P_{m}^{-1} F_{m}=J_{m} P_{m}^{-1}$. Note that due to the companion structure of matrix $F_{m}$, the first column of $P_{m}^{-1} F_{m}$ is the second column of $P_{m}^{-1}$ and so on until the last but one; hence the columns of $P_{m}^{-1} F_{m}$ are the columns of $P_{: \text {If }}^{-1}$ shifted one position and the last column of $P_{m}^{-1} F_{m}$ is $-q_{m, 0} b_{m}-q_{m, 1} \ddot{J_{m}} b_{m}-\ldots-q_{m, n, m-1} \tilde{J}^{\tilde{n}_{m-1}-1} b_{m}$, where $q_{m . i}$ denote the coefficients of $q_{m}(x)$.

On the right hand side, the product $\tilde{J}_{m} P_{m}{ }^{1}=\left[\tilde{J}_{m} b_{m}, \vec{J}_{m}^{2} b_{m}, \ldots, \tilde{J}_{m}^{t_{m} m} b_{m}\right]$ leads to the same column left shifting, whereas the last term is equal to the polynomial expression obtained for the last column of $P_{m}^{-1} F_{m}$ since $q_{m}\left(J_{m}\right)=0$ due to CayleyHamilton's theorem.

The use of $P^{-1}$ is illustrated with several examples in $[16,20,25]$, where no explicit proof of its structure is provided.

Let $B$ be a matrix with the same number of rows as $F_{1}$ and with $S$ columns. We write $B$ as

$$
B=\left(\begin{array}{cccc}
b_{1,1} & b_{1,2} & \cdots & b_{1, S} \\
b_{2,1} & b_{2,2} & \cdots & b_{2, S} \\
\vdots & \vdots & \ddots & \vdots \\
b_{M, 1} & b_{M, 2} & \cdots & b_{M, S}
\end{array}\right)
$$

in such a way that the product $P^{-1} B$ makes sense. If $b_{m, s}$ is the vector $\left(b_{m, s, 0}, b_{m, s, 1}, \cdots, b_{m, s, n, n-1}\right)^{\top}$, define $p_{m, s}(x)=$ $\sum_{\ell=0}^{n_{*}+r_{-}-1} b_{m, s, \ell} x^{\ell}$ and denote

$$
\mathbf{P}(x)=\left(\begin{array}{cccc}
p_{1,1}(x) & p_{1,2}(x) & \ldots & p_{1, S}(x) \\
p_{2,1}(x) & p_{2,2}(x) & \ldots & p_{2, S}(x) \\
\vdots & \vdots & \ddots & \vdots \\
p_{M, 1}(x) & p_{M, 2}(x) & \cdots & p_{M, S}(x)
\end{array}\right) .
$$

When addressing system controllability, for $1 \leq k \leq K$ we will have to consider a set $C_{k}$ of rows of $P^{-1} B$, which will correspond to the first $M_{k}$ rows of this polynomial matrix $P(x)$ evaluated at $\lambda_{k}$. 
We will establish a condition for the controllability of $(F, B)$, based on $Q_{m}(x)$, polynomials whose roots are eigenvalues of $F$ with exactly $m$ Jordan blocks in $J$. We will first show that $Q_{m}(x)$ can be obtained without knowing its roots.

We begin by constructing polynomials $\tilde{q}_{m}^{\prime}(x)$ for $1 \leq m \leq M$ whose roots are those of $q_{m}(x)$ but simple:

$$
\tilde{q}_{m}(x):=\frac{q_{m}(x)}{\operatorname{gcd}\left\{q_{m}(x), q_{m}^{\prime}(x)\right\}} .
$$

Since the roots of $q_{m}(x)$ are eigenvalues of $F$ with, at least, $m$ Jordan blocks, the roots of $Q_{m}$ are those of $q_{m}(x)$ which are not roots of $q_{m+1}(x)$. Thus, we define $Q_{M}(x)=\tilde{a}_{M}(x)$ and, for $1 \leq m \leq M-1$, we define $Q_{m}(x)=\frac{q_{m}(x)}{q_{m+1}\left({ }_{M}\right)}$. When no eigenvalue has exactly $m$ Jordan blocks, we obtain $Q_{m}(x) \in \mathbb{R} \backslash\{0\}$, which is coprime with any polynomial.

Now we are ready to state the following result:

Theorem 2. $(F, B)$ is controllable if and only if for each $1 \leq m \leq M$, if $Q_{m}(x) \notin \mathbb{R}$, then there exist order $m$ non-null minors of $\left.\mathbf{P}(x)\right|^{[m]}$ which are coprime together with $Q_{m}(x)$.

Proof. Based on Lemma 1 and Theorem 1, the pair $(F, B)$ is controllable if and only if, for $1 \leq k \leq K$,

$$
\operatorname{rank}\left(\left.\mathbf{P}\left(\lambda_{k}\right)\right|^{\left[M_{k}\right]}\right)=M_{k},
$$

or, equivalently, for $1 \leq k \leq K$ there is an order $M_{k}$ non-zero minor of $\left.\mathbf{P}\left(\lambda_{k}\right)\right|^{\left.\mid M_{k}\right]}$. The $K$ conditions of (8) can be reformulated as a set of alternative conditions for each $1 \leq m \leq M$, so that when no $\lambda_{k}$ has $m$ blocks, we have $Q_{m}(x) \in \mathbb{R} \backslash\{0\}$ and the theorem statement is trivially satisfied, whereas when several eigenvalues have exactly $m$ blocks they are considered jointly. In fact, if $\lambda_{k_{1}}, \lambda_{k_{2}} \ldots . \lambda_{k_{I}}$ satisfy that $M_{k_{1}}=M_{k_{2}}=\cdots=M_{k_{I}}$ then, the conditions (8) for these eigenvalues are equivalent to the existence, for $1 \leq \ell \leq L$, of an order $M_{k_{1}}$ non-zero minor of $\left.\mathbf{P}\left(\lambda_{\ell}\right)\right|^{\mid M_{k_{1}}}$, i.e., the existence of order $M_{k_{1}}$ non-zero minors of $\left.\mathbf{P}(x)\right|^{\left.\mid M_{k_{1}}\right]}$ which are coprime together with $Q_{M_{k}}(x)=\prod_{\ell-1}^{i}\left(x-\lambda_{k_{\ell}}\right)$.

Note that coprimality among any amount of polynomials can be checked without computing their roots, just resorting to the Euclidean division algorithm. This algorithm can be symbolically implemented, its only limitation coming from the available computer memory. In addition, the rational canonical form $F$ of a matrix $A$ and a nonsingular matrix $P$ such that $F=P^{-1} A P$ can be obtained by means of the computation of the Smith normal form of $x F-A$ and elementary row and column operations (see [26, Chapter 6]). In [27], an algorithm for determining the rational canonical form of a matrix is also provided, which employs computational symbolic algebraic manipulation packages, but it does not give a similarity matrix $P$. On the other hand, in [25] a numerical algorithm for obtaining both the rational canonical form $F$ of a matrix and its similarity matrix $P$ is proposed. This algorithm has polynomial cost and uses elementary row and column operations in order to obtain $F$ and $P$.

Example. Let us consider an order 31 square matrix $F=F_{1} \oplus F_{2} \oplus F_{3} \oplus F_{4}$, where for $k \in\{1,2,3,4\}$ each $F_{k}$ is the companion matrix corresponding to the characteristic polynomial $q_{k}(x)$ as listed below (for illustrative purposes, all polynomials are also shown in factorized form, although such form is not required for performing the required computations):

$$
\begin{aligned}
q_{1}(x)= & (x-1)^{4}(x+1)^{4}(x-2)^{3}(x+2)^{2} \\
= & x^{13}-2 x^{12}-12 x^{11}+24 x^{10}+54 x^{9}-108 x^{8}-116 x^{7}+232 x^{6} \\
& +129 x^{5}-258 x^{4}-72 x^{3}+144 x^{2}+16 x-32 . \\
q_{2}(x)= & (x-1)^{4}(x+1)^{3}(x-2)^{2} \\
= & x^{9}-5 x^{8}+5 x^{7}+11 x^{6}-21 x^{5}-3 x^{4}+23 x^{3}-7 x^{2}-8 x+4 . \\
q_{3}(x)= & (x-1)^{3}(x+1)^{2}(x-2) \\
= & x^{6}-3 x^{5}+6 x^{3}-3 x^{2}-3 x+2 . \\
q_{4}(x)= & (x-1)^{3} \\
= & x^{3}-3 x^{2}+3 x-1 .
\end{aligned}
$$

Then,

$$
\begin{aligned}
& \tilde{q}_{1}(x)=(x-1)(x+1)(x-2)(x+2)=x^{4}-5 x^{2}+4 \\
& \tilde{q}_{2}(x)=(x-1)(x+1)(x-2)=x^{3}-2 x^{2}-x+2 \\
& \widetilde{q}_{3}(x)=(x-1)(x+1)(x-2)=x^{3}-2 x^{2}-x+2 . \\
& \widetilde{q}_{4}(x)=x-1
\end{aligned}
$$

Finally,

$$
\begin{aligned}
& Q_{1}(x)=x+2 \\
& Q_{2}(x)=1
\end{aligned}
$$




$$
\begin{aligned}
& Q_{3}(x)=(x+1)(x-2)=x^{2}-x-2 \\
& Q_{4}(x)=x-1 .
\end{aligned}
$$

Let us consider a matrix $B$ having four columns (each one representing a controller) composed by the following submatrices:

$$
\begin{gathered}
\left.B\right|^{\{1, \ldots, 13)}=\left(\begin{array}{cccc}
6 & 66 & -48 & 42 \\
-60 & 13 & -49 & 19 \\
46 & -144 & 167 & -116 \\
155 & -12 & 121 & -46 \\
0 & 139 & -128 & 85 \\
-54 & 6 & -39 & 13 \\
51 & -148 & 173 & -120 \\
160 & -10 & 122 & -46 \\
-7 & 76 & -84 & 45 \\
6 & -7 & 10 & -6 \\
5 & -4 & 6 & -4 \\
5 & 2 & 1 & 0 \\
-1 & 3 & -4 & 2
\end{array}\right),\left.B\right|^{\{14, \ldots, 22\}}=\left(\begin{array}{cccc}
20 & 36 & -11 & 14 \\
6 & 18 & -2 & 1 \\
71 & -122 & 168 & -116 \\
186 & 38 & 105 & -24 \\
-35 & 101 & -122 & 64 \\
21 & -20 & 40 & -20 \\
11 & 14 & -3 & 6 \\
-12 & 7 & -15 & 7 \\
2 & -6 & 8 & -4
\end{array}\right) \\
\left.B\right|^{\{23, \ldots, 28\}}=\left(\begin{array}{cccc}
2 & 34 & -26 & 22 \\
-26 & 15 & -28 & 13 \\
38 & -120 & 138 & -100 \\
150 & 4 & 105 & -34 \\
-23 & 79 & -96 & 51 \\
3 & -9 & 12 & -6
\end{array}\right),\left.B\right|^{\{29, \ldots, 31\}}=\left(\begin{array}{cccc}
4 & & & \\
-40 & 5 & -27 & 24 \\
3 & -20 & 23 & -12
\end{array}\right)
\end{gathered}
$$

Therefore, matrix $\mathbf{P}(x)$ is given by:

$$
\begin{aligned}
p_{11}(x)= & -x^{12}+5 x^{11}+5 x^{10}+6 x^{9}-7 x^{8}+160 x^{7}+51 x^{6}-54 x^{5} \\
& +155 x^{3}+46 x^{2}-60 x+6 \\
p_{12}(x)= & 3 x^{12}+2 x^{11}-4 x^{10}-7 x^{9}+76 x^{8}-10 x^{7}-148 x^{6}+6 x^{5} \\
& +139 x^{4}-12 x^{3}-144 x^{2}+13 x+66 \\
p_{13}(x)= & -4 x^{12}+x^{11}+6 x^{10}+10 x^{9}-84 x^{8}+122 x^{7}+173 x^{6}-39 x^{5} \\
& -128 x^{4}+121 x^{3}+167 x^{2}-49 x-48 \\
p_{14}(x)= & 2 x^{12}-4 x^{10}-6 x^{9}+45 x^{8}-46 x^{7}-120 x^{6}+13 x^{5}+85 x^{4}-46 x^{3} \\
& -116 x^{2}+19 x+42 \\
p_{21}(x)= & 2 x^{8}-12 x^{7}+11 x^{6}+21 x^{5}-35 x^{4}+186 x^{3}+71 x^{2}+6 x+20 \\
p_{22}(x)= & -6 x^{8}+7 x^{7}+14 x^{6}-20 x^{5}+101 x^{4}+38 x^{3}-122 x^{2}+18 x+36 \\
p_{23}(x)= & 8 x^{8}-15 x^{7}-3 x^{6}+40 x^{5}-122 x^{4}+105 x^{3}+168 x^{2}-2 x-11 \\
p_{24}(x)= & -4 x^{8}+7 x^{7}+6 x^{6}-20 x^{5}+64 x^{4}-24 x^{3}-116 x^{2}+x+14 \\
p_{31}(x)= & 3 x^{5}-23 x^{4}+150 x^{3}+38 x^{2}-26 x+2 \\
p_{32}(x)= & -9 x^{5}+79 x^{4}+4 x^{3}-120 x^{2}+15 x+34 \\
p_{33}(x)= & 12 x^{5}-96 x^{4}+105 x^{3}+138 x^{2}-28 x-26 \\
p_{34}(x)= & -6 x^{5}+51 x^{4}-34 x^{3}-100 x^{2}+13 x+22 \\
p_{41}(x)= & 3 x^{2}-40 x+4 \\
p_{42}(x)= & -20 x^{2}+5 x+38 \\
p_{43}(x)= & 23 x^{2}-31 x-27 \\
p_{44}(x)= & -12 x^{2}+12 x+24
\end{aligned}
$$


The minors of matrix $\mathbf{P}(x)$ are:

$$
\begin{aligned}
\operatorname{det}(\mathbf{P}(x))= & 8 x^{23}+52 x^{22}+54 x^{21}-37 x^{20}-58 x^{19}-214 x^{18}-669 x^{17} \\
& -1097 x^{16}-1430 x^{15}-1864 x^{14}-2344 x^{13}-2338 x^{12}-2038 x^{11} \\
& -1913 x^{10}-1703 x^{9}-1170 x^{8}-580 x^{7}-295 x^{6}-82 x^{5}+108 x^{4} \\
& +94 x^{3}+20 x^{2} \\
= & x^{2}(x-2)(x+1)(x+5)(2 x-1)(2 x+1)^{2}\left(x^{3}+2\right)\left(x^{4}+1\right) \\
& \because\left(x^{2}+x+1\right)\left(x^{3}+x+1\right)\left(x^{3}+x^{2}+1\right),
\end{aligned}
$$

which does not have common factors with $Q_{4}(x)$.

$$
\begin{aligned}
\operatorname{det}\left(\left.\mathbf{P}(x)\right|_{[3]} ^{[1,2.3]}\right)= & 48 x^{22}+120 x^{21}+188 x^{20}+214 x^{19}-38 x^{18}-211 x^{17} \\
& -1216 x^{16}-2785 x^{15}-4330 x^{1}-6675 x^{13}-8270 x^{12} \\
& -10321 x^{11}-10366 x^{10}-8344 x^{9}-7476 x^{8}-7130 x^{7} \\
& -4308 x^{6}-580 x^{5}-298 x^{4}+192 x^{3}+1814 x^{2} \\
& +1420 x+312,
\end{aligned}
$$

which has no common roots with $Q_{3}(x)$. Regarding the other order 3 minors that can be constructed with the first 3 rows of $\mathbf{P}(x)$,

$$
\begin{aligned}
\operatorname{det}\left(\left.\mathbf{P}(x)\right|_{[3]} ^{[1,2.4]}\right)= & (x+1)(x-2)\left(8 x^{21}-4 x^{20}-74 x^{19}-175 x^{18}-290 x^{17}\right. \\
& -515 x^{16}-985 x^{15}-1154 x^{14}-1274 x^{13}-1445 x^{12} \\
& -1711 x^{11}-1358 x^{10}-128 x^{9}-777 x^{8}-768 x^{7}+59 x^{6} \\
& \left.+828 x^{5}+37 x^{4}+32 x^{3}+438 x^{2}-20 x-120\right), \\
\operatorname{det}\left(\left.\mathbf{P}(x)\right|_{[3]} ^{[1,3.4]}\right)= & (x+1)(x-2)\left(-8 x^{21}-28 x^{20}-30 x^{19}-61 x^{18}\right. \\
& -192 x^{17}-284 x^{16}-380 x^{15}-633 x^{14}-1004 x^{13} \\
& -1366 x^{12}-1162 x^{11}-1496 x^{10}-2099 x^{9}-1222 x^{8} \\
& -768 x^{7}-984 x^{6}-915 x^{5}+92 x^{4}+44 x^{3}-60 x^{2} \\
& +364 x+204), \\
\operatorname{det}\left(\left.\mathbf{P}(x)\right|_{[2,3]} ^{[2,3.4]}\right)= & (x+1)(x-2)\left(-8 x^{21}-12 x^{20}+18 x^{19}+27 x^{18}\right. \\
& -16 x^{17}+9 x^{16}+95 x^{15}-67 x^{14}-340 x^{13}-496 x^{12} \\
& -351 x^{11}-851 x^{10}-1582 x^{9}-667 x^{8}-446 x^{7}-904 x^{6} \\
& \left.-942 x^{5}+42 x^{4}-18 x^{3}-147 x^{2}+308 x+192\right),
\end{aligned}
$$

which, clearly, can be divided by $Q_{3}(x)$.

Since $Q_{2}(x)$ is a non-zero real number we do not need to check on it further. Finally, order 1 minors in the first row of $\mathbf{P}(x)$ are precisely its 4 elements. Since $x+2$ is not a factor of any element in the first row of $\mathbf{P}(x)$, all conditions of the theorem are satisfied and, therefore, the pair $(F, B)$ is controllable.

As mentioned above, standard control design problems deal with fixed matrices $A$ and $B$ which are pre-established by the system dynamics and/or actuator restrictions. In this context, rank condition (2) serves as an initial test for determining the a priori limitations of any controller to be designed, as well as a starting point to characterize further system properties $[1,28,29]$.

On the other hand, some alternative problems where design and control issues are jointly addressed may allow an arbitrary selection of the number and location of the actuators; this scenario is quite common when designing distributed control systems [9-11], and it has become specially relevant when addressing the controllability of systems modelled by complex networks $[12,13]$. In these alternative contexts, matrix $B$ becomes a design element, and the controllability condition serves as a design criterion. In the following section, this problem of selecting an appropriate matrix $B$ is addressed.

\section{Selection of matrix $B$ and number of required controllers}

As mentioned above, in contrast to classical control theory where the pair $(A, B)$ characterizing dynamics and actuator restrictions is given a priori, we consider now a new scenario which serves to model some alternative design/control problems. Specifically, we consider that only matrix $A$ is predefined (e.g, gathering structural information via a network model) whereas matrix $B$ (nodes of the network to act on) is considered as a design element to be selected so that system (1) satisfies the controllability condition. 
Obviously, if we select $B=I$ (meaning that $r=n$ ), then $(A, B)$ is controllable since $n=\operatorname{rank} B \leq \operatorname{rank} C(A, B) \leq n$ which implies $\operatorname{rank} C(A, B)=n$. Hence, the determination of a matrix $B$ which guarantees system controllability is, a priori, a trivial problem.

In practice, it may be very useful to find the simplest matrix $B$ (in a sense to be defined) that guarantees the controllability condition. A possible measure of simplicity might be $p$, the number of columns of matrix $B$, which represents the number of required actuators (components of input vector $u(t)=\left[u_{1}(t), \ldots, u_{p}(t)\right]$ ).

\subsection{Number of required controllers}

Let us consider $B \in \mathbb{R}^{n \times p}$ so that $\operatorname{rank} B=s<p$, then we can select $C=\left\{c_{1}, c_{2}, \ldots c_{s}\right\}$ linearly independent columns of $B$, so that the remaining columns can be obtained as a linear combination of them. Let us consider, without loss of generality, that $C=\{1, \ldots, s\}$ and let us denote $C^{\prime}=\{s+1, \ldots, p\}$. which means that $B=\left[\left.\left.B\right|_{C} B\right|_{C^{\prime}}\right]$ with $\operatorname{rank} B=\left.\operatorname{rank} B\right|_{C}=s$. Hence, each column of $\left.B\right|_{C^{\prime}}$ can be obtained via linear combination of the columns of $\left.B\right|_{C}$, meaning that we can write $B=\left.B\right|_{C}\left[I_{S} M\right]$ where $M$ gathers the coefficients of such linear combinations.

Therefore $B u(t)=\left.B\right|_{C}\left[I_{S} M\right] u(t)=\left.B\right|_{C} v(t)$ where $v(t)=\left[I_{S} M\right] u(t)=\left[v_{1}(t), \ldots, v_{s}(t)\right]^{\top}$ can be interpreted as a new control vector acting only on $\left.B\right|_{C}$ in an equivalent manner to $u(t)$ acting on $B$, and which is composed by only $s$ actuators, while preserving the same controllability condition

$$
\begin{aligned}
\operatorname{rank} C(A, B) & =\operatorname{rank}\left[\begin{array}{lll}
B A B & \cdots & A^{n-1} B
\end{array}\right] \\
& =\operatorname{rank}\left[\left.\left.\left.B\right|_{C} A B\right|_{C} \cdots A^{n-1} B\right|_{C}\right]=\operatorname{rank} C\left(A,\left.B\right|_{C}\right)
\end{aligned}
$$

Since we are interested in designing controllers with minimum number of external inputs, $B$ will be always selected so that it has maximum rank, and $\operatorname{rank} B$ will be the design index. Therefore, in the remaining of this section we address the problem of designing a matrix $B$ so that the system (1) is controllable and $B$ has a minimum number of columns (i.e., we want to determine the minimum number of required controllers for controlling system matrix $A$ ). Again, similarity matrices and canonical forms will prove to be useful for addressing this new problem:

Lemma 3. The minimum number of controllers required to control the system defined by matrix $A$ is the same as the minimum number of controllers required to control the system defined by any similar matrix $A \in S_{A}$.

Proof. Let us consider matrix $B$ having the minimum number of columns so that the pair $(A, B)$ is controllable. Let us consider $P$ such that $A=P^{-1} A P$. From Lemma 1 we know that the pair $\left(A, P^{-1} B\right)$ is also controllable, having $B$ and $P^{-1} B$ the same number of columns. Therefore $A$ can also be controlled with the same minimum number of required controllers to control $A$. The same reasoning can be applied by considering a matrix $B$ with minimum number of columns so that $(A, B)$ is controllable to conclude that such same number of controllers suffices to control $A$. Hence, the controllability of both $A$ and $A$ requires the same minimum number of controllers.

In particular, this is true when $\dot{A}=J_{A}$ and $\ddot{A}=F_{A}$, the Jordan or Frobenius forms associated with $A$, respectively.

\subsection{System matrix in Jordan canonical form}

We present now the main result concerning the minimum number of controllers (i.e., the minimum number of columns in $B$ ) required for the pair $(A, B)$ to be controllable. Such number is determined by the following theorem:

Theorem 3. Consider a matrix A so that the corresponding Jordan and Frobenius canonical forms are given in (3) and (4) respectively. Then, the minimum number of columns of $B$ for the pair $(A, B)$ to be controllable is $M=\max \left\{M_{k}: 1 \leq k \leq K\right\}$.

Proof. Let us consider that the pair $(A, B)$ is controllable and that $B$ has less than $M$ columns. By Lemma $\left.3 U_{A}, B^{\prime}\right)$ is also controllable for $B^{\prime}=P^{-1} B$ having also less than $M$ columns. Then for any selection of rows $R$ of $B^{\prime}$ we have that rank $\left.B^{\prime}\right|^{R}<M$ and considering condition (6) of Theorem 1 we have that for $C_{1}$, the selection of columns of $J$ corresponding to the first column of each Jordan block $J_{1 . m}$ associated with $\lambda_{1}$, we have $\left.\operatorname{rank} B^{\prime}\right|^{C_{1}}<M=M_{1}$ which concludes that the pair $\left(J\right.$, $\left.B^{\prime}\right)$ is not controllable. Therefore the number of required controllers (columns of $B$ ) must be greater or equal than $M$.

We now construct a matrix $B$ with $M$ columns which will satisfy the controllability condition. Let us consider $B$ with $M$ columns so that

$$
\left.B\right|^{C_{k}}=\left(I_{M_{k}} \mid O_{k}\right), \quad 1 \leq k \leq K
$$

where $C_{k}$, for $1 \leq k \leq K$, is the selection of columns of $J_{A}$ corresponding to the first column of each Jordan block $J_{k m}$, $I_{M_{k}}$ denotes the identity matrix of order $M_{k}$, and $0_{k}$ is the null matrix with $M_{k}$ rows and $M-M_{k}$ columns (if $M=M_{k}$ then $\left.B\right|^{C_{k}}=I_{M_{k}}$ ). By Theorem 1, the pair $\left(J_{A}, B\right)$ is controllable, no matter how we choose the undefined remaining rows of $B$. Hence, by Lemma 1 matrix $A$ can be controlled with $M$ controllers and this is the minimum number of controllers to guarantee its controllability.

Note that the proof can also be developed by resorting to the Frobenius canonical form associated with $A$ given by (4). We can construct a matrix $B$ with $M$ columns satisfying

$$
\left.B\right|^{C}=I_{M:}
$$


so that $C$ is the selection of columns of $F_{A}$ corresponding to the first column of each Vandermonde block $F_{m}$. It is straightforward to show that the pair $\left(F_{A}, B\right)$ is controllable, no matter how we choose the undefined remaining rows of $B$. Remember that $F_{m} \sim J_{m}$ which only gathers at most a single Jordan block of each eigenvalue (with geometric multiplicity 1 ), so that it can be controlled with a single input [30].

Remark 1. Although the statement of Theorem 3 is simple and intuitive, it has not been found explicitly stated in the literature. The authors believe that it could also be proved using fundamental results concerning canonical forms presented in [16-21], even though these works are framed in dealing with a given $B$ matrix, and no minimization problem is addressed therein.

Some of the algorithms to compute the Jordan and/or the Frobenius canonical forms do also provide the corresponding transition matricx $P$ (e.g., see [26]). Hence, since the proof of Theorem 3 provides a means to construct a matrix $B^{\prime}$ (associated with the selected canonical form) satisfying the minimum number of columns condition, one can compute matrix $B=P B^{\prime}$ associated with the original system matrix $A$, which will also satisfy this same condition. From there on, a controller can be designed for $(A, B)$ following some of the many known control design algorithms [31,32]. In any case, the applicability of Theorem 3 relies on the computational cost and the robustness of the available algorithms for obtaining the Jordan and/or the Frobenius forms [22-26,33].

\section{Number of required dedicated controllers}

So far, the design of matrix $B$ has not taken into account the number of nonzero elements in each column $B_{j}$. Each nonzero element of $B_{j}$ indicates that the external input $j$ directly acts on the state variable corresponding to such element. Since the system state variables may be physically different, sometimes one may not be able to implement a real actuator for simultaneously operating on several of them. Hence, it makes sense to consider the case when actuators affect only one state variable. We call dedicated controllers to those external inputs entering a unique state variable. Note that if matrix $B$ characterizes dedicated controllers, each column $B_{j}$ has only one nonzero element which we assume to be unitary, without loss of generality. Obviously different actuators will operate on different variables so that the number of columns of $B$ will remain being the same as $\operatorname{rank} B$.

Given a system represented by matrix $A$, let us call $N_{d c}(A)$ the minimum number of dedicated controllers required for controlling such system. The following theorem establishes the range of the number of dedicated controllers any matrix $A$ may require for its controllability.

Theorem 4. Let $A \in \mathbb{R}^{n \times n}$ and let $J_{A}$ and $F_{A}$ be its associated Jordan and Frobenius canonical forms given in (3) and (4) respectively. Then

$$
M=\max \left\{M_{k}: 1 \leq k \leq K\right\}=N_{d c}\left(F_{A}\right) \leq N_{d c}(\dot{A}) \leq N_{d c}\left(J_{A}\right)=\sum_{k=1}^{K} M_{k} .
$$

Proof. Considering (10), we can select zero values for the remaining terms of matrix $B$, so that $F_{A}$ can be controlled with the minimum number of controllers, $M$, already in dedicated form (i.e., $N_{d G}\left(F_{A}\right)=M$ ). Since $A$ cannot be controlled with less than $M$ controllers, necessarily $M \leq N_{d c}(A)$.

Let us consider now the upper bound associated with $J_{A}$. By Theorem 1 , rank $\left.B\right|^{C_{k}}=M_{k}$ and $B$ needs to have $M_{k}$ non-zero rows for each $k \in\{1, \ldots, K\}$. Note that $C_{k}$ are by definition disjoint sets, so that $B$ will have at least $\sum_{i}^{i i} M_{k}$ non-zero rows (and hence, such number of non-zero elements). Since we are considering dedicated controllers (each column can only have one non-zero element) $B$ must have necessarily at least $\sum_{k=1}^{K} M_{k}$ non-zero columns. We will prove that $\sum_{i=1}^{\ddot{\prime}} M_{k}$ controllers are sufficient to guarantee dedicated controllability by constructing an appropriate matrix $B$. To satisfy the conditions of Corollary 1 , we can just choose $D_{j}=\left[1+\sum_{i-1}^{j-1} M_{i}, \sum_{i-1}^{j} M_{i}\right]$ and $\left.B\right|_{C_{k}} ^{l, j}=\delta_{k_{j} j} I_{M_{k}}$, for $1 \leq k, j \leq K\left(\bar{\delta}_{i j}\right.$ being the Kronecker delta). By setting the rest of rows of $B$ to zero we have dedicated controllers satisfying (7), the number of columns of $B$ being $\sum_{k=1}^{K} M_{k}$.

Let us finally consider the second inequality. Note that if matrix $B$ characterizes $s$ dedicated controllers, $B$ has only one nonzero unitary element per column and it has $s$ nonzero rows with one nonzero unitary element each, which can be gathered in $R=\left\{r_{1}, \ldots, r_{s}\right\}$, so that $\left.\operatorname{rank} B\right|^{R}=s=|R|$, the cardinal of $R$. Based on Lemma 1 , controlling $A$ via $B$ is equivalent to controlling $J_{A}$ with some matrix $P^{-1} B=\bar{P}^{-\hat{i}} \dot{I}_{R}$. the outcome of selecting the $s$ corresponding columns of a corresponding regular similarity matrix $P^{-1}$. Let us define $C_{k}$ as in Theorem 1 . By Lemma $1, \mathcal{C}\left(J . P^{-1}\right)=n$ and by Corollary 1 , for $1 \leq k \leq K$ we can find a selection $D_{k}$ of columns of $P^{-1} \mid C_{k}$ such that rank $\left.P^{-1}\right|_{\bar{\nu}_{k}} ^{-L_{k}}=M_{k}$. Then, following the reasoning of proof of Corollary 1 , $D=\bigcup_{k=1}^{K} D_{k}$ is a selection of columns of $P^{-1}$ such that rank $C\left(J,\left.P^{-1}\right|_{D}\right)=n$. Moreover,

$$
|D|=\left|\bigcup_{k=1}^{K} D_{k}\right| \leq \sum_{k=1}^{K}\left|D_{k}\right|=\sum_{k=1}^{K} M_{k}=N_{d c}\left(U_{h}\right) \text {. }
$$

Hence, we can control $J_{A}$ with matrix $\left.P^{-1}\right|_{D}$ which was proven to be equivalent to selecting $|D| \leq N_{d c}(J)$ dedicated controllers for matrix $A$. This implies that $N_{d c}(A) \leq N_{d c}(J)$. 
Note that the Jordan form represents maximal disaggregation on invariant subspaces (i.e., decoupling); hence, we need as many dedicated $B_{j}$ columns of matrix $B$ as Jordan blocks of $J$. On the other hand, the Frobenius form represents minimal disaggregation on invariant subspaces (i.e., coupling), since controllable subspaces are as larger as possible, generated from the iteration of a single vector.

In some specific cases, such as when the associated Jordan form of $A$ has $n$ distinct eigenvalues, $N_{d c}(A)$ can be obtained as the minimum number of columns of the transformation matrix $P$ which must be selected so that the corresponding submatrix has non-zero rows. Note that each column of $P$ can be "activated" by a corresponding column in $B$ with a single nonzero element, so that $P B$ is such submatrix which guarantees that $(J, P B)$ is controllable (every row has a non-zero entry).

The following simple example illustrates the role of the Jordan versus Frobenius structures for determining the number of required dedicated controllers.

\subsection{A simple example}

Let us consider system matrix $A$ and its corresponding canonical forms

$$
A=\left(\begin{array}{cccc}
0 & 0 & 0 & 0 \\
1 & 1 & 0 & 0 \\
0 & 0 & -1 & 0 \\
0 & 0 & 1 & -1
\end{array}\right) \sim J=\left(\begin{array}{cccc}
0 & 0 & 0 & 0 \\
0 & 1 & 0 & 0 \\
0 & 0 & -1 & 0 \\
0 & 0 & 1 & -1
\end{array}\right) \sim F=\left(\begin{array}{cccc}
0 & 0 & 0 & 0 \\
1 & 0 & 0 & 1 \\
0 & 1 & 0 & 1 \\
0 & 0 & 1 & -1
\end{array}\right)
$$

so that $A$ is a block diagonal matrix whose corresponding submatrices come in Frobenius and Jordan forms respectively. Considering general controllability, by Theorem $3, A$ can be controlled with a single controller (e.g., $B=(1,0,1,0)^{T}$ would serve such purpose). On the other hand, for dedicated controllability, Theorem 4 states that $1=N_{d c}(F) \leq N_{d c}(A) \leq N_{d c}(J)=3$. Note that $A$ cannot be controlled by a single dedicated controller since, being block diagonal, defines a decoupling between the first two and the last two variables; it is easy to check that $A$ can be controlled by matrix

$$
B=\left(\begin{array}{ll}
1 & 0 \\
0 & 0 \\
0 & 1 \\
0 & 0
\end{array}\right)
$$

which proves that $N_{d c}(A)=2$.

\section{Concluding remarks}

Given a system state dynamics, the determination of the minimum number of required controllers provides new insight for establishing the number and location of the actuators in the system control design process. The bounds provided for the case when dedicated controllers are needed open the way to further research issues in this area.

For systems with pre-established control inputs (i.e., fixed matrix $B$ ), the proposed alternative characterizations of the controllability condition, based on the Jordan or Frobenius canonical forms, may overcome some of the computational limitations (in terms of cost and ill conditioning) of algorithms for checking Kalman's general rank condition.

The results developed in this paper have direct analogs for the system observability dual problem.

\section{Acknowledgement}

The present work has been partially supported by project MTM2015-67396-P of Ministerio de Economía y Competitividad, Spain.

\section{References}

[1] E.G. Gilbert, Controllability and observability in multivariable control systems, J. Soc. Ind. Appl. Math. Ser. A Control 1 (2) (1963) $128-151$.

[2] E. Krèindler, P. Sarachik, On the concepts of controllability and observability of linear systems, IEEE Trans. Autom. Contrò 9 (2) (1964) 129-136, doi:10.1109/TAC.1964.1105665.

[3] D. Luenberger, Introduction to Dynamic Systems: Theory, Models, and Applications, Wiley, 1979.

[4] M. Vidyasagar, Nonlinear Systems Analysis, 42, SIAM, 2002.

[5] R.C. Dorf, Modern Control Systems, Addison-Wesley Longman Publishing Co, Inc., 1995.

[6] C.-T. Chen, Linear System Theory and Design, oxford University Press, Inc., 1995.

[7] B. Friedland, Control System Design: An Introduction to State Space Methods, McGraw-Hill, Inc., 1986.

[8] R.E. Kalman, et al., Contributions to the theory of optimal control, Bol. Soc. Mat. Mexicana 5 (2) (1960) 102-119.

[9] K. Morris, Lineat-quadratic optimal actuator location, IEEE Trans. Autom, Control 56 (1) (2011) 113-124.

[10] N. Darivandi, K. Morris, A. Khajepour, An algorithm for LQ optimal actuator location, Smart Mater. Struct. 22 (3) (2013) 035001.

[11] D. Kasinathan, K. Morris, $H_{\infty}$-optimal actuator location, IEEE Trans. Autom. Control 58 (10) (2013) 2522-2535, doi:10,1109/TAC,2013.2266870,

[12] Y.-Y. Liu, J.-J. Slotine, A.-L. Barabasi, Controllability of complex networks, Nature 473 (7346) (2011) 167-173.

[13] P.J. Zufiria, L. Ubeda-Medina, C. Herrera-Yagüe, I. Barriales-Valbuena, Mathematical foundations for efficient structural controllability and observability analysis of complex systems, Math. Probl. Eng. 2014 (2014).

[14] H.Y. Cheung, T.C. Kwok, L.C. Lau, Fast matrix rank algorithms and applications, J. ACM (JACM) 60 (5) (2013) 31

[15] F. Le Gall, Powers of tensors and fast matrix multiplication, in: Proceedings of the Thirty Ninth International Symposium on Symbolic and Algebraic Computation, ACM, 2014, pp. 296-303. 
[16] J.H. Wilkinson, The Algebraic Eigenvalue Problem, 87, Clarendon Press Oxford, 1965.

[17] D. Luenberger, Canonical forms for linear multivariable systems, IEEE Trans. Autom. Control 12 (3) (1967) $290-293$.

[18] N. Sinha, P. Rozsa, Some canonical forms for linear multivariable systems, Int. J. Control 23 (6) (1976) $865-883$.

[19] J. Maroulas, S. Barnett, Applications of the comrade matrix to linear multivariable systems theory, Int. J. Control 28 (1) (1978) $129-145$.

[20] H.W. Turnbull, A.C. Aitken, An Introduction to the Theory of Canonical Matrices, Courier Corporation, 2004.

[21] W.A. Wolovich, Linear Multivariable Systems, 11, Springer Science \& Business Media, 2012.

[22] G.H. Golub, J.H. Wilkinson, Ill-conditioned eigensystems and the computation of the jordan canonical form, SIAM Rev. 18 (4) (1976) 578-619.

[23] J.W. Demmel, Applied Numerical Linear Algebra, 56, SIAM, 1997.

[24] Z. Bai, J. Demmel, J. Dongarra, A. Ruhe, H. van der Vorst, Templates for the Solution of Algebraic Eigenvalue Problems: A Practical Guide, SIAM, 2000.

[25] P. Ozello, Calcul exact des formes de Jordan et de Frobenius d'une matrice, Ph.D. thesis, Universit Scientifique Technologique et Medicale de Grenoble, 1987.

[26] L. Hogben, Handbook of Linear Algebra, Chapman and Hall/CRC, 2007.

[27] J.S. Devitt, R.A. Mollin, The rational canonical form of a matrix, Int. J. Math. Math. Sci. 9 (2) (1986) 253260.

[28] A. Hamdan, A. Nayfeh, Measures of modal controllability and observability for first-and second-order linear systems, J. Guid. Control Dyn. 12 (3) (1989) $421-428$.

[29] B. Molinari, A strong controllability and observability in linear multivariable control, IEEE Trans. Autom. Control 21 (5) (1976) 761-764.

[30] A. Portal, P.J. Zufiria, Controlling systems with a single actuator, in: Proceedings of International Conference on Logistics, Informatics and Service Science, LISS, Springer, 2015, pp. 1197-1202.

[31] V.L. Mehrmann, The Autonomous Linear Quadratic Control Problem: Theory and Numerical Solution, 163, Springer, 1991.

[32] F. Aliev, V.B. Larin, Optimization of Linear Control Systems: Analytical Methods and Computational Algorithms, CRC Press, 1998.

[33] B. Kagstrom, A. Ruhe, An algorithm for numerical computation of the jordan normal form of a complex matrix, ACM Trans. Math. Softw. (ToMS) 6 (3) (1980) $398-419$. 